\title{
Correction: Protective role of p53 in skin cancer: Carcinogenesis studies in mice lacking epidermal p53
}

\section{Angustias Page, Manuel Navarro, Cristian Suarez-Cabrera, Josefa P. Alameda, M. Llanos Casanova, Jesús M. Paramio, Ana Bravo, Angel Ramirez}

Present: Due to an error in grant support.

Correct: The updated grant support are given below. The author sincerely apologizes for this error.

Original article: Oncotarget. 2016; 7(15):20902-18. doi: 10.18632/oncotarget.7897.

\section{GRANT SUPPORT}

This research was supported partially by funds from Fondo Europeo de Desarrollo Regional (FEDER) and by grants from the Ministry of Economy and Competitiveness of Spain (FIS Exp PI14/01403 from the Instituto de Salud Carlos III to AR) and by grants from the Spanish government (CIEM13-4E-1944 to AR; PI13/02580 to MLC and SAF2012-34378 and SAF- 2015-66015-R to JMP from the Ministerio de Economía y Competitividad; RD12/0036/0009 -to JMP- from the Instituto de Salud Carlos III and S2011/BMD2470 -to JMP- from the Comunidad Autónoma de Madrid). 